Article

\title{
Design and Implementation of a New Wireless Carotid Neckband Doppler System with Wearable Ultrasound Sensors: Preliminary Results
}

\author{
Ilseob Song ${ }^{1}$, Jongmin Yoon ${ }^{1}$, Jinbum Kang ${ }^{1}$, Min Kim ${ }^{1}$, Won Seuk Jang ${ }^{2}{ }^{\oplus}$, Na-Young Shin ${ }^{3}$ \\ and Yangmo Yoo ${ }^{1,4, *}$ \\ 1 Department of Electronic Engineering, Sogang University, Seoul 04107, Korea; ilseob@sogang.ac.kr (I.S.); \\ jongminyoon@u.sogang.ac.kr (J.Y.); jbkang@sogang.ac.kr (J.K.); drxmin@gmail.com (M.K.) \\ Department of Medical Engineering, Yonsei University, Seoul 06229, Korea; WS.JANG@yuhs.ac \\ 3 Department of Radiology, The Catholic University of Korea, Seoul 06591, Korea; c0na79@gmail.com \\ 4 Department of Biomedical Engineering, Sogang University, Seoul 04107, Korea \\ * Correspondence: ymyoo@sogang.ac.kr; Tel.: +82-02-705-4731
}

Received: 17 April 2019; Accepted: 24 May 2019; Published: 29 May 2019

check for updates

Featured Application: Continuous monitoring of cerebrovascular and cardiovascular diseases.

\begin{abstract}
Noninvasive monitoring of blood flow in the carotid artery is important for evaluating not only cerebrovascular but also cardiovascular diseases. In this paper, a wireless neckband ultrasound Doppler system, in which two 2.5-MHz ultrasonic sensors are utilized for acquiring Doppler signals from both carotid arteries, is presented for continuously evaluating blood flow dynamics. In the developed wireless neckband Doppler system, the acquired Doppler signals are quantized by 14-bit analog-to-digital-converters running at $40 \mathrm{MHz}$, and pre-processing operations (i.e., demodulation and clutter filtering) are performed in an embedded field programmable gate array chip. Then, these data are transferred to an external smartphone (i.e., Galaxy S7, Samsung Electronics Co., Suwon, Korea) via Bluetooth 2.0. Post-processing (i.e., Fourier transform and image processing) is performed using an embedded application processor in the smartphone. The developed carotid neckband Doppler system was evaluated with phantom and in vivo studies. In a phantom study, the neckband Doppler system showed comparable results with a commercial ultrasound machine in terms of peak systolic velocity and resistive index, i.e., $131.49 \pm 3.97$ and $0.75 \pm 0.02$ vs. $131.89 \pm 2.06$ and $0.74 \pm 0.02$, respectively. In addition, in the in vivo study, the neckband Doppler system successfully demonstrated its capability to continuously evaluate hemodynamics in both common carotid arteries. These results indicate that the developed wireless neckband Doppler system can be used for continuous monitoring of blood flow dynamics in the common carotid arteries in point-of-care settings.
\end{abstract}

Keywords: carotid ultrasonography; neckband; wearable ultrasound; point-of-care

\section{Introduction}

Noninvasive monitoring of blood flow in the common carotid arteries is important for evaluating not only cerebrovascular diseases [1,2] but also cardiovascular diseases [3,4]. Among various imaging modalities, Doppler ultrasonography is a popular tool for measuring blood flow velocity and its variability in the common carotid arteries [5-7]. In carotid Doppler ultrasonography, spectral broadening with an elevated peak systolic velocity (PSV) on a pulsed-wave spectral Doppler waveform is correlated with the degree of carotid stenosis [8-11]. In addition, carotid Doppler ultrasonography can provide quantitative functional measurements of blood flow, e.g., the resistive index, $R I$, which is a hemodynamic parameter reflecting local wall extensibility and related vascular resistance [12-14]. 
Moreover, if Doppler ultrasonography can continuously measure the blood flow of the common carotid artery, increased intracranial pressure can be assessed in patients with head trauma, stroke, brain tumor, or hydrocephalus [15-17]. Additionally, by intraoperatively monitoring emboli during endovascular or surgical procedure of aorta, heart, or carotid arteries, the risk of potential stroke and brain injury can be reduced [18,19]. However, utilizing a conventional cart-based Doppler ultrasound system is challenging for continuous assessment of the common carotid artery due to its limited accessibility in terms of cost and size.

Recently developed portable and handheld ultrasound imaging systems feature substantially reduced sizes and costs for bedside and intraoperative procedures, but they are still inconvenient for use in continuously monitoring the common carotid artery. To lower operator dependency, in the last two decades, various types of wearable ultrasound Doppler device prototypes have been investigated [20,21]. For monitoring blood flow in the common carotid artery, the 'Doppler Necklace' system, in which an ultrasound array transducer was mounted to acquire blood flow signal, was proposed. However, it was not sufficient to validate the concept of the 'Doppler Necklace' since theoretical analysis and simulation results were limited [20-24]. Another wearable carotid ultrasound device, in which two 128-element linear array probes were mounted similarly to the original design, was also proposed. Although this carotid ultrasound device can successfully visualize common carotid arteries to measure their diameters, it cannot evaluate blood flow velocity and its variability [24]. In this paper, a new wireless carotid wearable ultrasound Doppler system, in which two single-element ultrasonic sensors are mounted in the neckband for acquiring Doppler signals from both carotid arteries, is proposed for continuously measuring blood flow velocities and functional index parameters (e.g., RI). In the developed wireless carotid neckband ultrasound Doppler system, the acquired Doppler signals are quantized, and pre-processing operations (i.e., demodulation and clutter filtering) are performed on a field programmable gate array chip (Sparatan-6 LX150, Xilinx Inc., San Jose, CA, USA). Then, these data are transferred to an external smartphone via Bluetooth 2.0. Post-processing (i.e., Fourier transform and image processing) is performed using an embedded application processor in the smartphone. The developed wireless carotid wearable ultrasound Doppler system prototype was evaluated with a string phantom and in vivo studies.

\section{Materials and Methods}

\subsection{Design of a Wireless Carotid Wearable Ultrasound Doppler System}

Figure 1 shows the functional block diagram of the proposed wireless carotid neckband ultrasound Doppler system. As shown in Figure 1, the two single-element transducers for the left and right common carotid arteries are used to transmit and receive ultrasound waves, and then the received radio-frequency $(\mathrm{RF})$ ultrasound signals are quantized in analog-to-digital conversion (A/D conversion).

In the pre-processing step, the quantized RF data are first transmitted with low-voltage differential signaling (LVDS) protocol and then converted as parallel data in the serial-to-parallel conversion, and the multiplexing (MUX) is used for selecting one of the two single-element transducers. After serial-to-parallel conversion and multiplexing, the quantized ultrasound RF data undergo high-pass filtering before quadrature demodulation to remove DC components, i.e., DC rejection filtering. To extract complex baseband data (i.e., in-phase and quadrature components), quadrature demodulation is performed, which involves multiplying the DC-rejected RF data by a carrier-frequency reference signal and then low-pass filtering the result of the multiplication [25]. After quadrature demodulation, complex baseband data are accumulated over the depth within a sample volume by using a window function (e.g., hanning). Lastly, wall filtering is applied to remove the clutter originating from slowly moving vessel walls and/or tissue movement [26]. After wall filtering, complex-based band data are transferred to an external smartphone for post-processing via Bluetooth 2.0. 


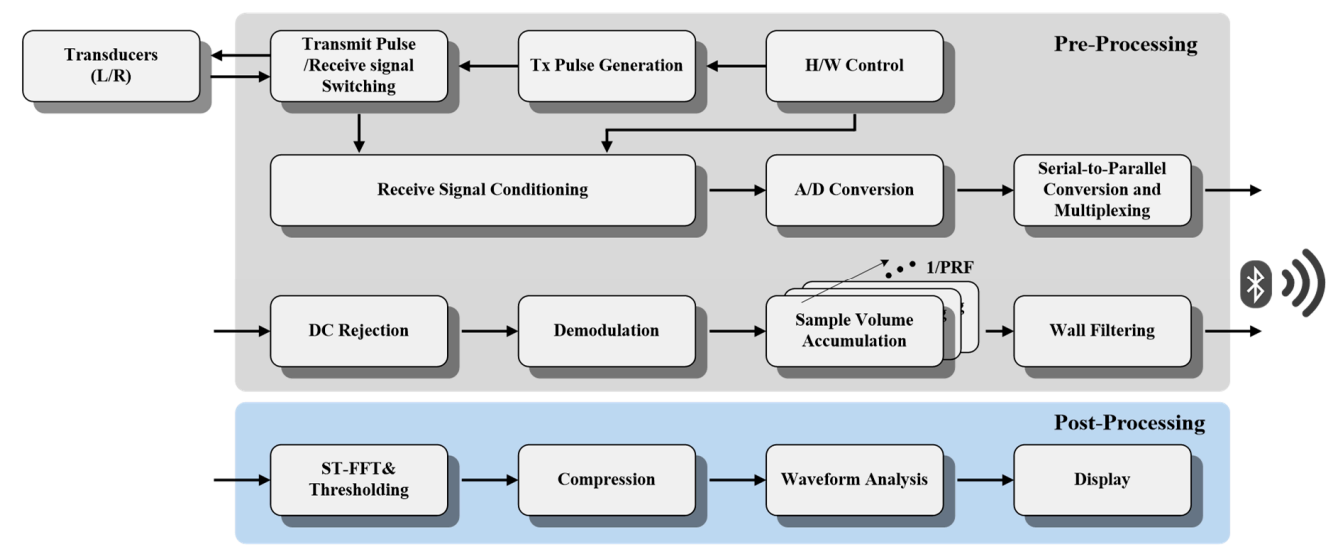

Figure 1. Functional block diagram of the wireless carotid wearable ultrasound Doppler system; ST-FFT: Short-time fast Fourier transform; PRF: Pulse-repetition frequency.

In the post-processing step, short-time fast Fourier transform (ST-FFT) is applied for generating Doppler spectra representing the changing velocity throughout the cardiac cycle and the distribution of velocities in the common carotid arteries $[27,28]$. Then, the amplitude of the Doppler spectra is computed, and a thresholding operation is performed to remove the noise. The amplitude of the Doppler spectra undergoes compression, in which a predesigned nonlinear curve (e.g., log) is applied to adjust its dynamic range for enhanced visualization. Finally, a spectral Doppler waveform is generated, and some quantitative measurements are obtained (e.g., PSV and RI).

Figure 2 shows the overall system design of the proposed wireless carotid wearable ultrasound Doppler device supporting the functional blocks described in Figure 1. As shown in Figure 2, two 2.5-MHz single-element transducers are connected to a multi-channel pulser chip with a transmit/receive switch, and the received signals are sent to a multi-channel ultrasound analog front-end (AFE) chip consisting of low-noise amplifiers (LNAs), time-gain compensators (TGCs), programmable-gain amplifiers (PGAs), and 14-bit ADCs running at $40 \mathrm{MHz}$ with a LVDS protocol.

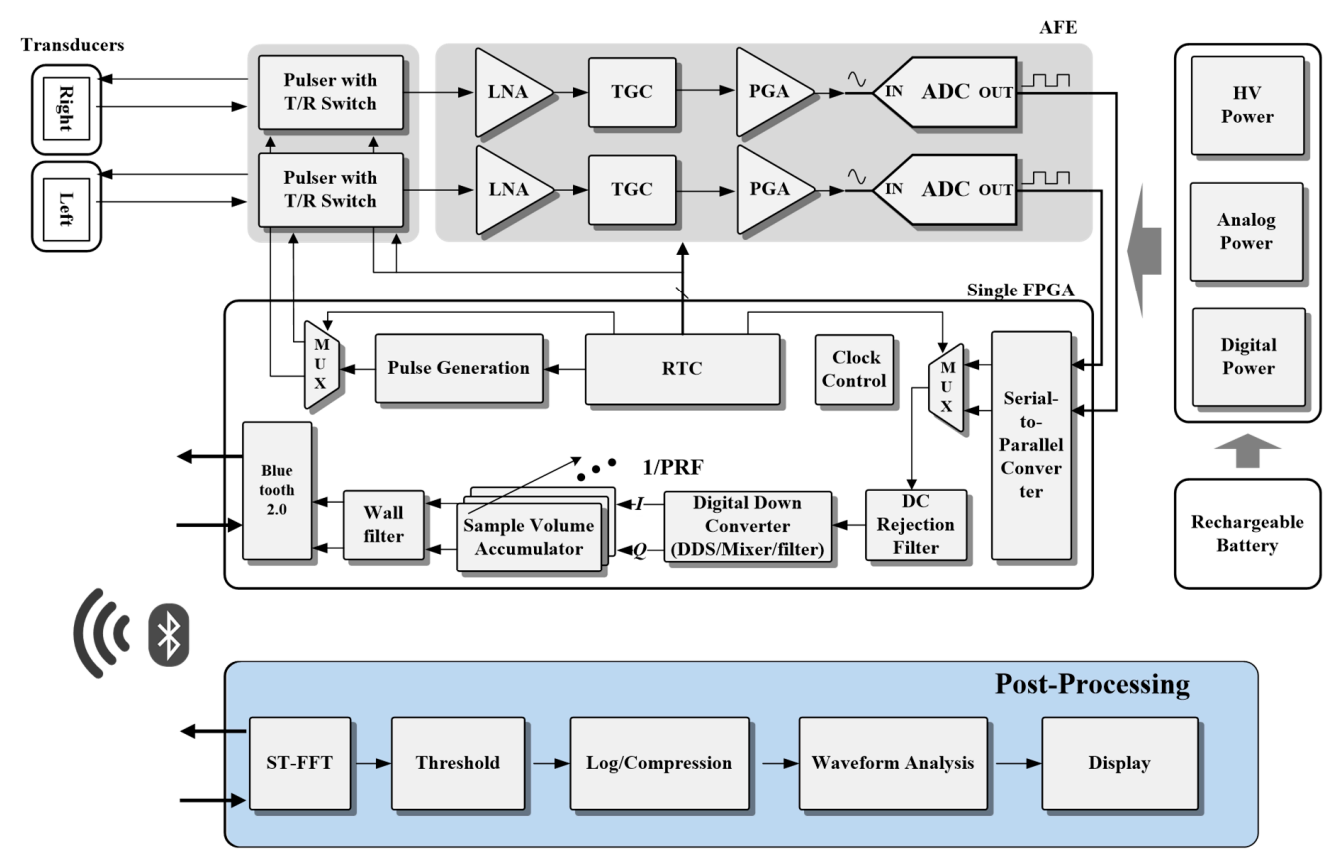

Figure 2. Overall system design of the wireless carotid wearable ultrasound Doppler device; LNA: Low-noise amplifier; TGC: Time-gain compensator; PGA: Programmable gain amplifier; ADC: Analog-to-digital converter; RTC: Real-time controller; MUX: Multiplexer. 
An FPGA chip (Sparatan-6 LX150, Xilinx Inc., San Jose, CA, USA) is used for generating electrical pulses and is controlled by a real-time controller (RTC) based on the required PRF, and the chip is also used for performing pre-processing operations on the quantized ultrasound RF data. For pre-processing, a 128-tap finite impulse response (FIR) filter is employed to remove DC components in DC rejection filtering, and a direct digital synthesizer, mixer and digital filter are implemented for quadrature demodulation. After quadrature demodulation, $40-\mathrm{MHz}$ in-phase and quadrature components in complex baseband data are separately accumulated with a hanning window whose length is determined from the selected sample volume size in the sample volume accumulator, and these accumulated complex baseband data are gathered with a given PRF (e.g., $4 \mathrm{kHz}$ ). To remove clutter components, in wall filtering, a 128-tap FIR filter is applied. After wall filtering, a commercial Bluetooth 2.0 module (i.e., HC-06, Guangzhou HC Information Technology Co., Ltd., Guangzhou, China) is used for wirelessly transmitting the complex baseband data to the external smartphone (Galaxy S7, Samsung Electronics Co., Suwon, South Korea). In the smartphone, a complex FFT with varying window sizes (e.g., 128) is first performed; then, the amplitude of the output of the FFT operation is calculated. These operations were implemented with the Java running on OpenGL ES 3.0. To remove the noise, the threshold operation based on the comparison between the input data and the predetermined value is performed. Log-compression is applied to lower the dynamic range of the processed data, and the PSV and RI are measured. Lastly, these values are displayed along with a spectral Doppler waveform on the screen of the smartphone. An in-house power module is used for supplying necessary analog and digital power to the designed wireless carotid wearable ultrasound Doppler system.

\subsection{Experimental Setup}

The performance of the wireless carotid wearable ultrasound Doppler device prototype was evaluated with phantom and in vivo studies. In the phantom study, as shown in Figure 3, a Model 043 Doppler string phantom (CIRS Inc., Norfolk, VA, USA) was used. In the string phantom, as shown in Figure 3, a filament was attached to a series of pulley wheels and a drive wheel was controlled by an electronic controller producing steady or pulsatile movement of the filament. In the phantom study, the pre-programmed pulsatile flow (i.e., adult common carotid mode) was selected and the filament is accordingly moved. The movement of the filament produced a flow around the filament. A $2.5-\mathrm{MHz}$ bipolar electrical pulse with 20 cycles was generated, and the peak-to-peak voltage applied to the ultrasound transducer was $40 \mathrm{Vpp}$. The PRF was set to $4 \mathrm{kHz}$. The PSV and end diastolic velocity $(E D V)$ were first measured, and then, the $R I$ value was computed by

$$
R I=\frac{P S V-E D V}{P S V} .
$$

The $R I$ values measured by the developed prototype were compared with those from a commercial rack-based ultrasound machine (Accuvix V10, Samsung Medison Co., Seongnam, South Korea).

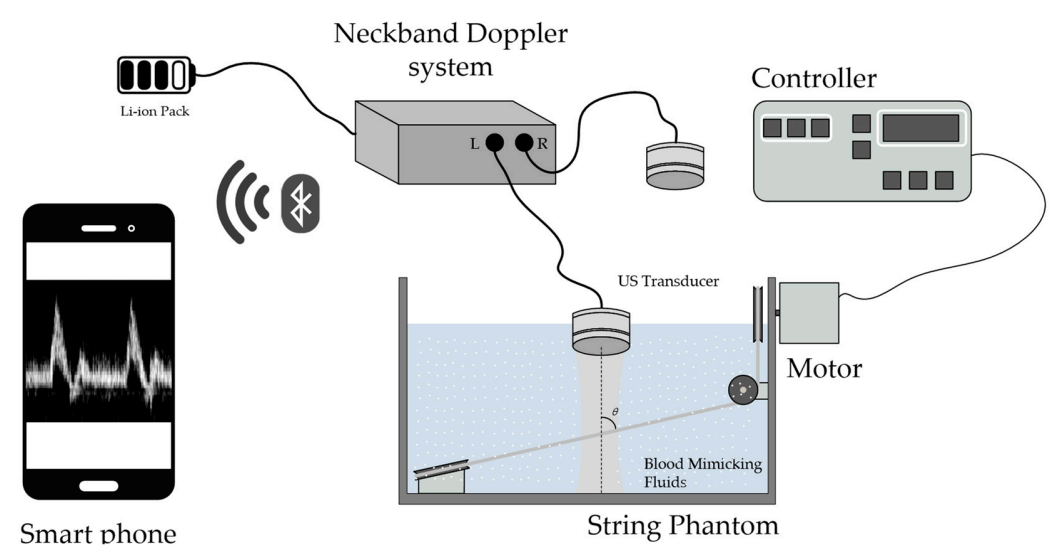

Figure 3. Experimental setup for the Doppler string phantom. 
In addition, to demonstrate the feasibility of the wireless carotid wearable ultrasound Doppler device for clinical use, an in vivo study was conducted on a healthy volunteer to measure the PSV and $R I$ values under the institutional review board (IRB) approval. The same parameters used in the phantom study were applied to the in vivo study.

\section{Results and Discussion}

\subsection{Prototype of a Wireless Carotid Wearable Ultrasound Doppler System}

The developed prototype of the wireless carotid neckband ultrasound Doppler system is shown in Figure 4. It consists of two single-element ultrasound transducers mounted on the neckband, two coaxial cables for the transducer connection, an ultrasound pre-processing module, a Bluetooth module, a power module, a battery pack, and a smartphone.

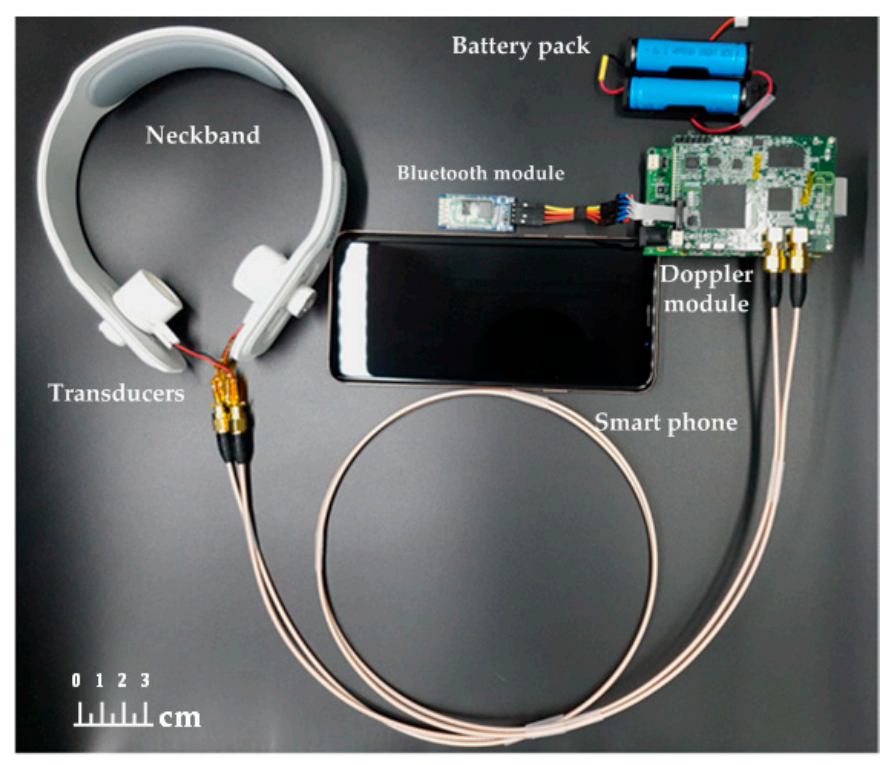

(a)

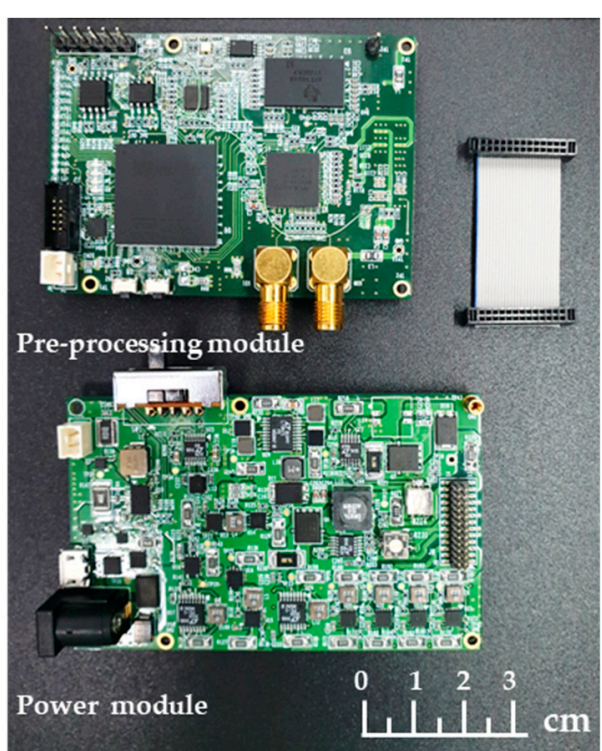

(b)

Figure 4. (a) Prototype of the wireless carotid wearable ultrasound Doppler system and (b) the pre-processing module and the power module.

The neckband is used for holding the transducers, and their positions can be adjusted to find the proper locations of the common carotid arteries. The two coaxial cables are used to connect the two $2.5-\mathrm{MHz}$ transducers to the pre-processing module. Figure $4 \mathrm{~b}$ shows the pre-processing module and the power module. The sizes of the pre-processing module and the power module are $70 \mathrm{~mm}$ (width) $\times$ $50 \mathrm{~mm}$ (height) and $80 \mathrm{~mm}$ (width) $\times 50 \mathrm{~mm}$ (height), respectively. The two modules are interconnected via a 26-pin flat cable. The developed wireless carotid wearable ultrasound Doppler system can be operated by two serially connected, $3.7 \mathrm{~V}, 750 \mathrm{mAh}$ Li-ion batteries, and can be continuously operated for approximately $1.5 \mathrm{~h}$.

\subsection{Phantom Study}

Figure $5 a, b$ shows the Doppler spectrograms obtained from the commercial rack-based ultrasound machine (i.e., Accuvix V10) and the developed wireless carotid wearable ultrasound Doppler system prototype, respectively. From visual assessment, the developed prototype successfully visualizes the pulsatile flow waveform similarly to the commercial rack-based ultrasound machine. The systolic and diastolic phases are clearly differentiated, and comparable spectral broadening is observed in the two spectrograms. Moreover, two systems show parabolic velocity profiles, as shown in the right side of the Figure $5 \mathrm{a}, \mathrm{b}$, at the peak systolic phase indicated by white dotted lines. Compared to the 
commercial rack-based ultrasound machine, the developed prototype presents more noise spikes and lower frequency resolution (i.e., a blocky representation of the velocity).
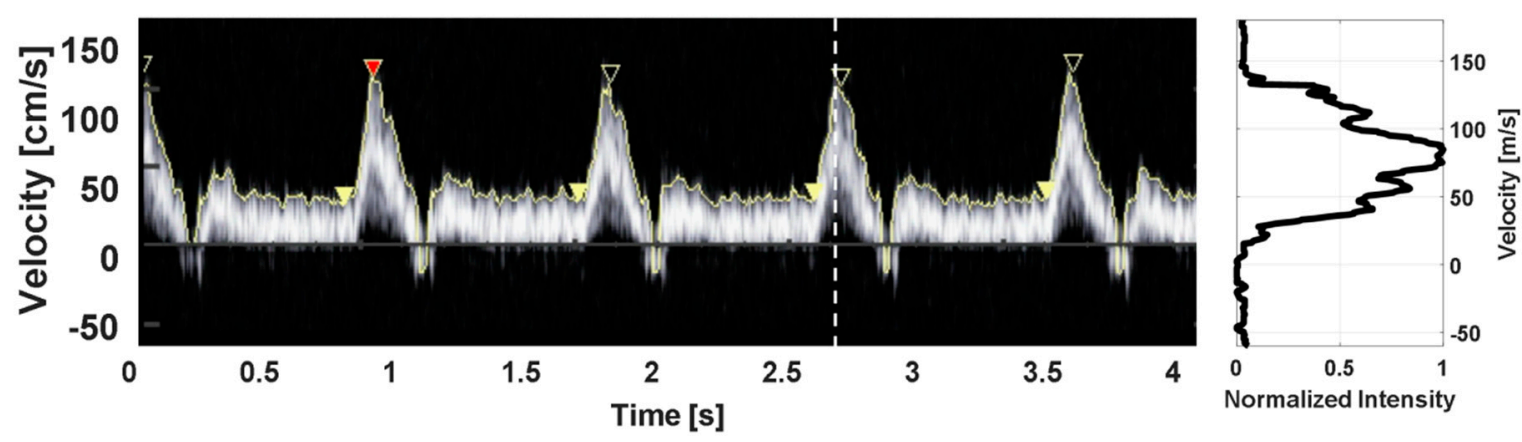

(a)

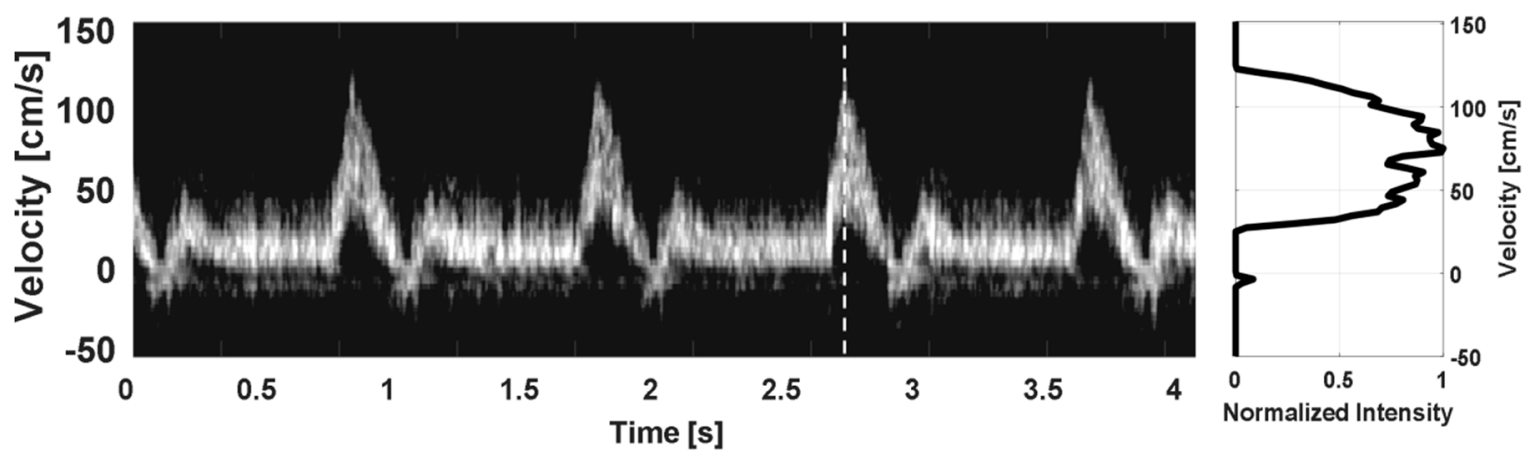

(b)

Figure 5. Doppler spectrogram obtained from (a) the commercial rack-based ultrasound machine (i.e., Accuvix V10, Samsung Medison Co., Seongnam, South Korea) and (b) the developed wireless carotid wearable ultrasound Doppler system prototype $(\mathrm{PRF}=4 \mathrm{KHz})$. The velocity profile at a peak systolic phase, indicated by a white dotted line, is given in the right side of each Doppler spectrogram.

The PSV and RI values from the commercial machine and the developed prototype were measured, and the results are summarized in Table 1. As listed in Table 1, the PSV and RI values for the commercial machine and the prototype were $131.49 \pm 3.97$ and $0.75 \pm 0.02$ vs. $131.89 \pm 2.06$ and $0.74 \pm 0.02$, respectively. There is no statistically significant difference in the measured PSV and RI values between the commercial rack-based machine and the wearable Doppler device prototype $(p=0.66$ and $p=0.70$, respectively). In addition, the velocity component at a specific time was also similary measured in the same manner as shown in Figure 5.

Table 1. The measured peak systolic velocity (PSV) and resistive index (RI) values (mean \pm std) from the commercial rack-based Accuvix V10 machine and the developed wireless carotid wearable ultrasound Doppler system prototype.

\begin{tabular}{cccc}
\hline Parameter & Accuvix V10 $(\boldsymbol{n}=\mathbf{1 0})$ & $\begin{array}{c}\text { Wearable Doppler } \\
\text { Prototype }(\boldsymbol{n}=\mathbf{1 0})\end{array}$ & $\boldsymbol{p}$-Value \\
\hline$P S V[\mathrm{~cm} / \mathrm{s}]$ & $131.49 \pm 3.97$ & $131 \pm 2.06$ & 0.64 \\
$R I$ & $0.75 \pm 0.02$ & $0.74 \pm 0.02$ & 0.70 \\
\hline
\end{tabular}

Boxplots of the measured PSV and RI values are also shown in Figure 6. As indicated in Table 1, the commercial rack-based machine and the wearable Doppler device prototype show comparable results. 


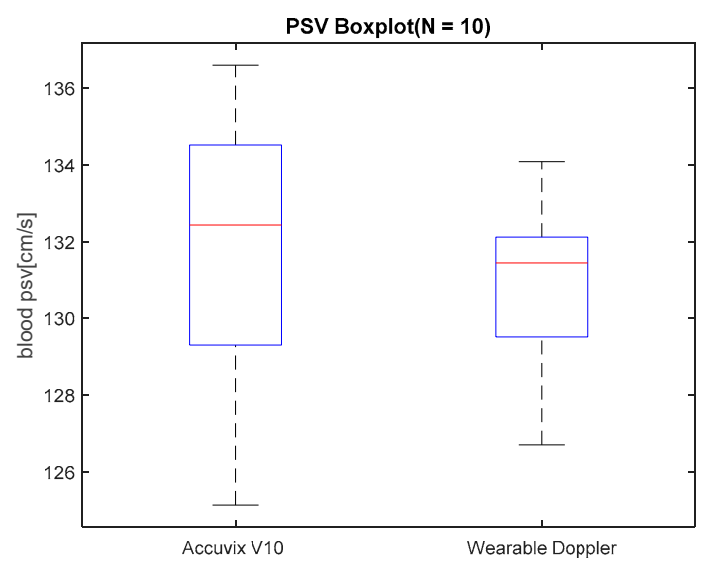

(a)

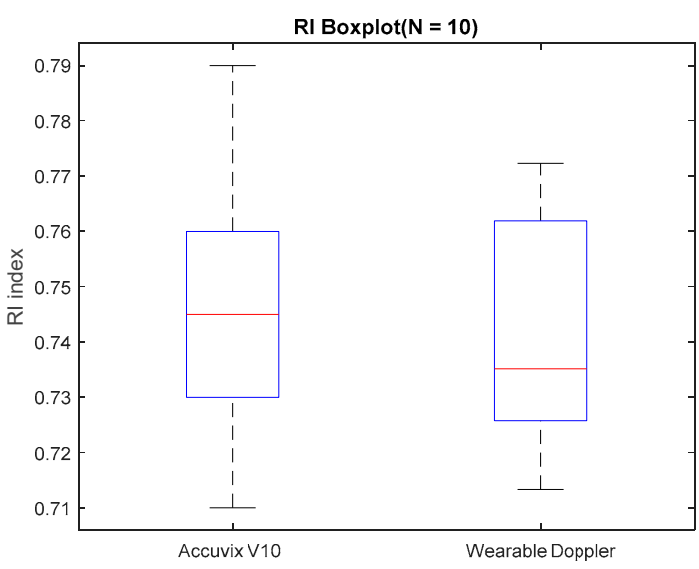

(b)

Figure 6. Boxplots of (a) PSV and (b) RI values from the commercial rack-based ultrasound machine (Accuvix V10) and the developed wireless carotid wearable ultrasound Doppler system prototype.

In the developed system, the system PRF setting determines the measurable maximum speed of blood flow. For example, with the default setting (i.e., $\mathrm{PRF}=4 \mathrm{kHz}$ ), the developed prototype is capable of measuring up to $150.0 \mathrm{~cm} / \mathrm{s}$ in common carotid arteries, as shown in Figure $5 \mathrm{~b}$. With PRF $=6 \mathrm{kHz}$, the measurable speed is extended up to $225.0 \mathrm{~cm} / \mathrm{s}$ without aliasing. Figure 7 shows the obtained result when using PRF $=6 \mathrm{kHz}$ from the string phantom for an acquisition time of $10 \mathrm{~s}$.

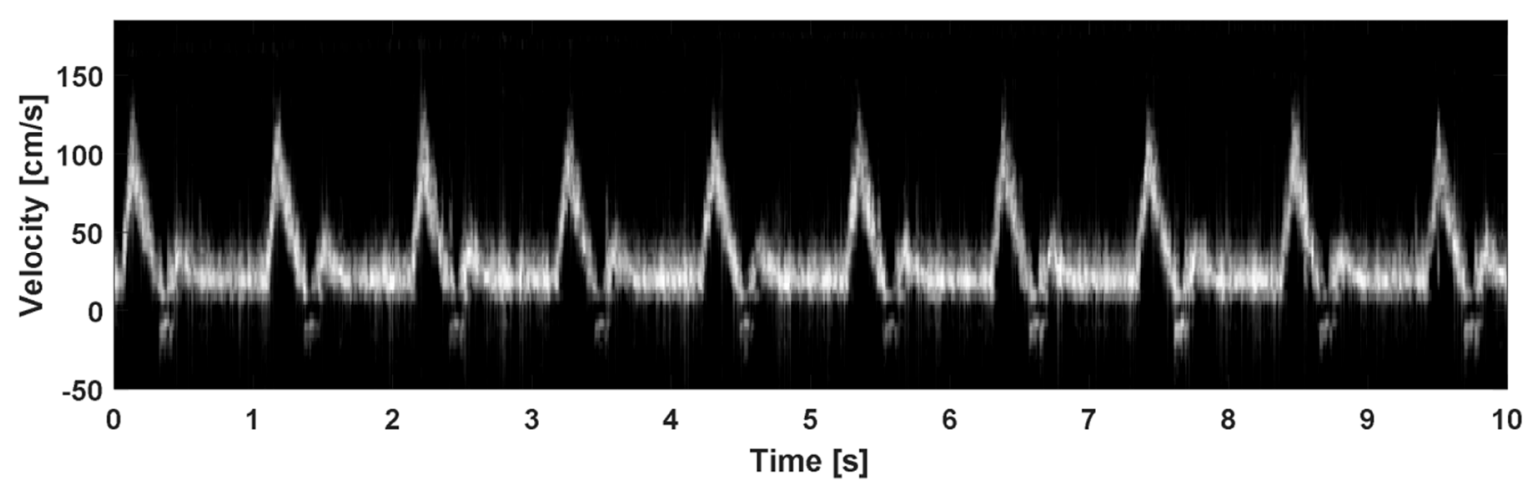

Figure 7. Doppler spectrogram obtained from the developed wireless carotid wearable ultrasound Doppler system prototype $(\mathrm{PRF}=6 \mathrm{KHz})$.

\subsection{In Vivo Study}

Figure 8 shows the placement of the developed wireless neckband carotid wearable ultrasound Doppler system prototype. As shown in Figure 8, the developed neckband ultrasound Doppler system can be placed on the neck so that blood flow dynamics from the common carotid arteries can be continuously monitored. It must be noted that, in the in vivo study, the location of the volunteer's common carotid artery was found by adjusting the position of the transducer mounted in the neckband. Before placing the neckband ultrasound Doppler system, an acoustic gel was first applied to the two ultrasonic sensors for providing sufficient contact to the skin. Each measurement took $3 \mathrm{~min}$. 

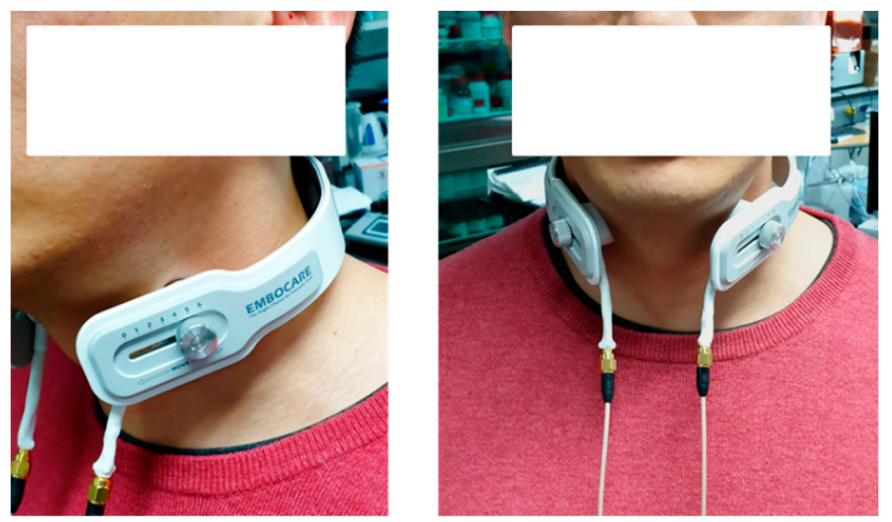

Figure 8. Placement of the wireless neckband carotid wearable ultrasound Doppler system prototype.

Figure 9 shows the spectrogram of the left and right common carotid arteries from a volunteer using the developed wireless wearable ultrasound Doppler system prototype. Comparable to the phantom study, the pulsatile waveform from the common carotid artery was successfully reconstructed, and the systolic and diastolic phases are clearly differentiated. In addition, the dicrotic notch is clearly visualized.

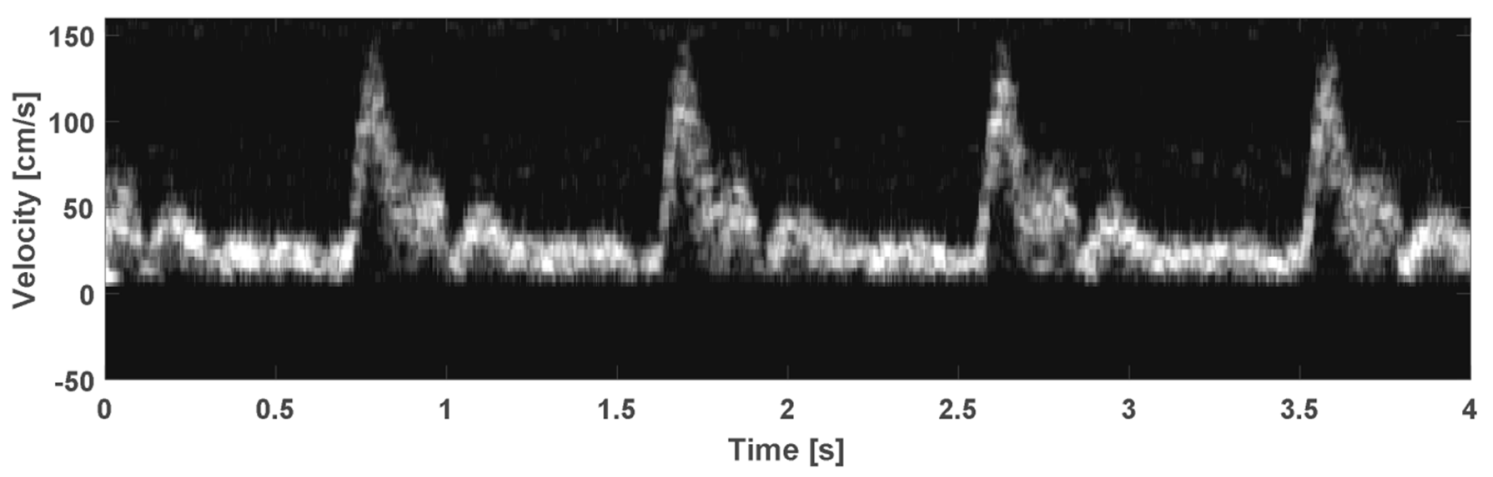

(a)

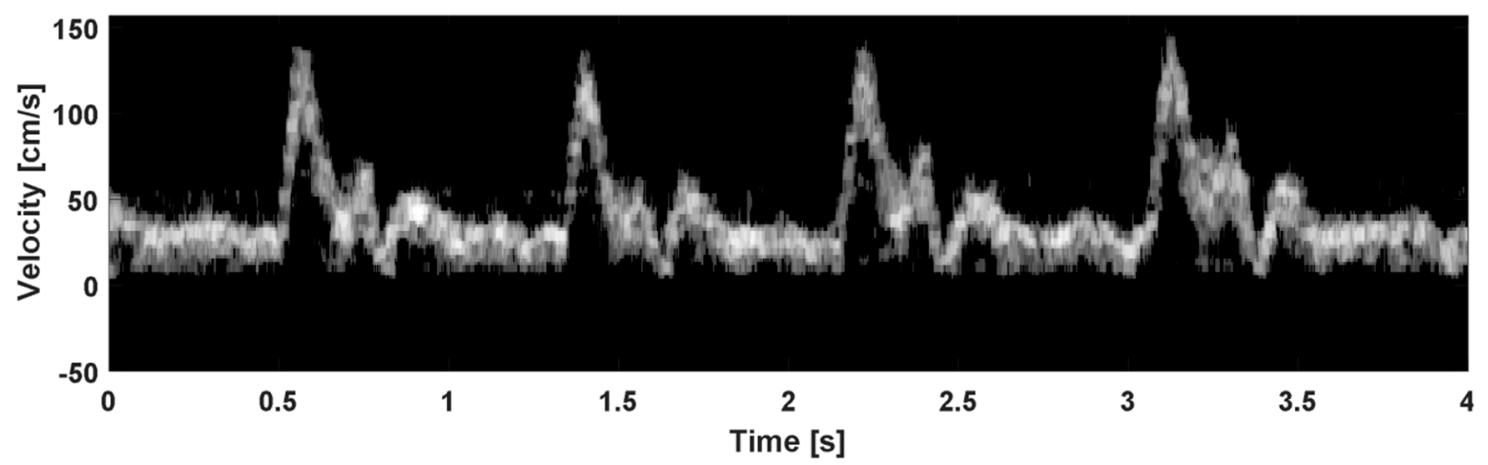

(b)

Figure 9. Spectrograms of (a) the left common carotid artery and (b) the right common carotid artery from a volunteer using the wireless carotid wearable ultrasound Doppler system prototype.

The PSV and RI values are measured and summarized in Table 2. As listed in Table 2, the developed wireless carotid wearable ultrasound Doppler system prototype successfully measured the PSV and RI values from the left and right common carotid arteries, i.e., $137.09 \pm 4.82$ and $0.83 \pm 0.01$ vs. $138.21 \pm$ 6.73 and $0.81 \pm 0.02$, respectively. 
Table 2. The measured peak systolic velocity (PSV) and resistive index (RI) values (mean \pm std) from the left and right common carotid arteries from a volunteer using the developed wireless carotid wearable ultrasound Doppler system prototype.

\begin{tabular}{ccc}
\hline Parameter & Left $(\boldsymbol{n}=\mathbf{1 0})$ & Right $(\boldsymbol{n}=\mathbf{1 0})$ \\
\hline$P S V[\mathrm{~cm} / \mathrm{s}]$ & $137.09 \pm 4.82$ & $138.21 \pm 6.73$ \\
$R I$ & $0.83 \pm 0.01$ & $0.81 \pm 0.02$ \\
\hline
\end{tabular}

\subsection{Discussion}

A wireless neckband ultrasound Doppler system was proposed for continuous monitoring of the common carotid arteries, and its prototype was developed. From the phantom study, the developed prototype showed comparable results with a commercial rack-based ultrasound machine (Accuvix V10) in terms of the visual assessment of the spectrogram and the measured values (i.e., PSV and $R I)$. However, the image quality of the spectrogram from the developed prototype is worse than that from the commercial rack-based ultrasound machine. This may be caused mainly by electrical noise, a less-optimized ultrasound Doppler signal and the image processing parameters (e.g., the window size in the FFT and the thresholding value). In addition, limited image enhancement functional blocks are only implemented in the developed prototype to lower the computational complexity of the post-processing running in the application processor of the smartphone. Thus, the image quality in the developed prototype can be further enhanced by adding some image processing blocks applied in ultrasound B-mode imaging. For example, the spikes of the spectrogram shown in Figures $5 \mathrm{~b}$ and $8 \mathrm{can}$ be reduced by applying 2D median filtering (e.g., $3 \times 3$ ) and 1 D lateral smoothing filtering (e.g., 3-tap low-pass FIR filter). The sharpness of the spectrogram can be improved by using edge enhancement filtering (e.g., unsharp masking [29]). However, due to the limited computational resource in the smart-phone, the computational requirement must be carefully analyzed for implementing these images processing algorithms for the developed wireless neckband ultrasound Doppler system.

Currently, the position of the two ultrasound transducers mounted in the neckband is adjusted to find the location of the common carotid arteries, and then the sample volume is manually positioned to obtain the proper spectrogram of the common carotid arteries. The location of the sample volume may be automatically determined by analyzing the pulsatile characteristics in the amplitudes of the complex baseband data acquired from the predetermined depths. For example, if the sample volume size is determined as $5 \mathrm{~mm}$ and the overlap ratio is $50 \%$, there are seven candidates of the optimal sample volume location when the scanning depth is from $10 \mathrm{~mm}$ to $30 \mathrm{~mm}$. The pulsatility from the seven candidate locations can be measured, and the optimal sample volume position can be determined when the pulsatile index reaches a maximum.

The proposed wireless carotid neckband ultrasound Doppler system was preliminarily evaluated with phantom and in vivo experiments. For use in clinical settings, further clinical evaluation is necessary. Once the proposed wearable Doppler device is clinically validated, it can be used during coronary artery bypass surgery where the detection of emboli is critical to lower the risks of stroke and brain injury. Additionally, it can be used for continuously monitoring hemodynamics in common carotid arteries to monitor intracranial pressure, which can be increased in patients with head trauma, stroke, brain tumor, or hydrocephalus. Moreover, the proposed wireless carotid neckband ultrasound Doppler system can be used as a monitoring tool for symptomatic or asymptomatic patients with cerebrovascular and/or cardiovascular diseases, as shown in Figure 10. Risk indicators can be continuously measured and sent to a server for archiving and data analysis and for predicting abnormalities in advance. Deep learning can be applied to identify proper indicators of cerebrovascular and/or cardiovascular diseases by using the Doppler spectrum archived in a patient database. Alternatively, the spectrogram of symptomatic or asymptomatic patients wearing the wireless carotid neckband ultrasound Doppler device can be fed to the server, and if a risk associated with cerebrovascular and/or cardiovascular 
diseases is indicated, this information can be directly sent to the patient or healthcare providers for proper intervention.

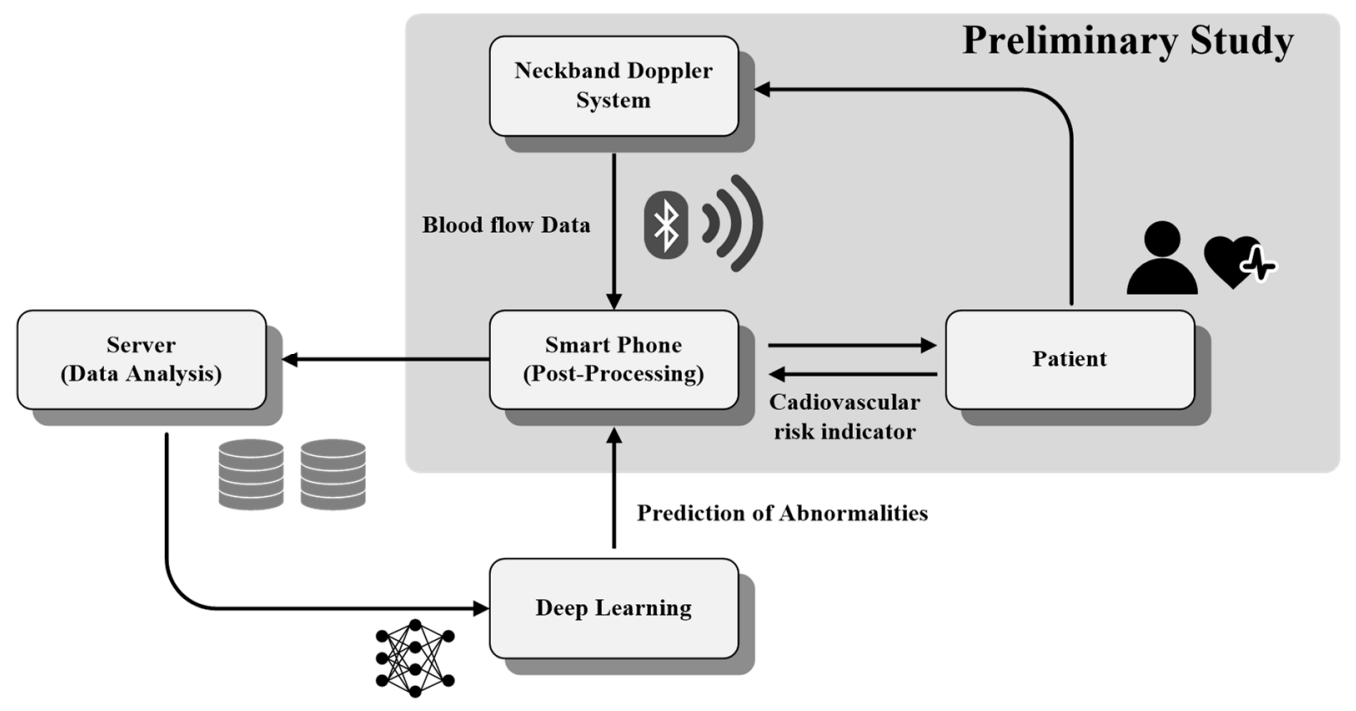

Figure 10. Prediction model of cerebrovascular and/or cardiovascular diseases by using the wireless carotid neckband ultrasound Doppler system and Deep Learning.

\section{Conclusions}

In this paper, a wireless carotid neckband ultrasound Doppler system was proposed for continuously monitoring blood flow dynamics in the common carotid arteries. From the phantom study, the developed wireless Doppler device prototype showed comparable results with a commercial rack-based ultrasound machine in terms of visual assessment and measured parameters. In addition, in the preliminary in vivo study, the spectrograms of the common carotid arteries of a volunteer were successfully reconstructed and the peak systolic velocities and resistive indexes were reliably calculated. These results indicate that the developed wireless neckband Doppler system can be used for continuous monitoring of blood flow dynamics in the common carotid arteries in point-of-care settings after further clinical investigation.

Author Contributions: Conceptualization, I.S. and J.Y.; investigation, I.S., J.Y., M.K., and J.K.; writing-original draft preparation, I.S.; writing - review and editing, N.-Y.S., W.S.J., and Y.Y.; project administration, Y.Y.; funding acquisition, N.-Y.S. and Y.Y.

Funding: This work was supported by the Bio \& Medical Technology Development Program of the National Research Foundation of Korea (NRF) funded by the Ministry of Science and ICT (NRF-2015M3A9D7067240).

Conflicts of Interest: The authors declare no conflict of interest. The funders had no role in the design of the study; in the collection, analyses, or interpretation of data; in the writing of the manuscript; or in the decision to publish the results.

\section{References}

1. Rankin, R.N.; Hutton, L.; D'Alotto, C. Carotid Doppler evaluation in cerebrovascular disease. Can. Med. Assoc. J. 1984, 130, 672-673. [PubMed]

2. Johnston, K.W.; deMorais, D.; Kassam, M.; Brown, P.M. Cerebrovascular assessment using a Doppler carotid scanner and real-time frequency analysis. J. Clin. Ultrasound. 1981, 9, 443-449. [CrossRef] [PubMed]

3. Sanguigni, V.; Gallù, M.; Strano, A. Incidence of carotid artery atherosclerosis in patients with coronary artery disease. Angiology 1993, 44, 34-38. [CrossRef]

4. Kazum, S.; Eisen, A.; Lev, E.I.; Iakobishvili, Z.; Solodky, A.; Hasdai, D.; Kornowski, R.; Mager, A. Prevalence of Carotid Artery Disease among Ambulatory Patients with Coronary Artery Disease. Isr. Med. Assoc. J. 2016, 18, 100-103. 
5. Keller, H.; Meier, W.; Yonekawa, Y.; Kumpe, D. Noninavasive angiography for the diagnosis of carotid artery disease using Doppler ultrasound (carotid artery Doppler). Stroke 1976, 7, 354-363. [CrossRef] [PubMed]

6. Lindegaard, K.F.; Bakke, S.J.; Grolimund, P.; Aaslid, R.; Huber, P.; Nornes, H. Assessment of intracranial hemodynamics in carotid artery disease by transcranial Doppler ultrasound. J. Neurosurg. 1985, 63, 890-898. [CrossRef]

7. Bishop, C.C.; Powell, S.; Rutt, D.; Browse, N.L. Transcranial Doppler measurement of middle cerebral artery blood flow velocity: A validation study. Stroke 1986, 17, 913-915. [CrossRef]

8. Garth, K.E.; Carroll, B.A.; Sommer, F.G.; Oppenheimer, D.A. Duplex ultrasound scanning of the carotid arteries with velocity spectrum analysis. Radiology 1983, 147, 823-827. [CrossRef] [PubMed]

9. Brown, P.M.; Johnston, K.W.; Kassam, M.; Cobbold, R.S. A critical study of ultrasound Doppler spectral analysis for detecting carotid disease. Ultrasound Med. Biol. 1982, 8, 515-523. [CrossRef]

10. Sheldon, C.D.; Murie, J.A.; Quin, R.O. Ultrasonic doppler spectral broadening in the diagnosis of internal carotid artery stenosis. Ultrasound Med. Biol. 1983, 9, 575-580. [CrossRef]

11. Jacobs, N.M.; Grant, E.G.; Schellinger, D.; Byrd, M.C.; Richardson, J.D.; Cohan, S.L. Duplex carotid sonography: Criteria for stenosis, accuracy, and pitfalls. Radiology 1985, 154, 385-391. [CrossRef]

12. Frauchiger, B.; Schmid, H.P.; Roedel, C.; Moosmann, P.; Staub, D. Comparison of carotid arterial resistive indices with intima-media thickness as sonographic markers of atherosclerosis. Stroke 2001, 32, 836-841. [CrossRef]

13. Staub, D.; Meyerhans, A.; Bundi, B.; Schmid, H.P.; Frauchiger, B. Prediction of cardiovascular morbidity and mortality: Comparison of the internal carotid artery resistive index with the common carotid artery intima-media thickness. Stroke 2006, 37, 800-805. [CrossRef]

14. Bude, R.O.; Rubin, J.M. Relationship between the resistive index and vascular compliance and resistance. Radiology 1999, 211, 411-417. [CrossRef] [PubMed]

15. Schneider, R.C.; Lemmen, L.J. Traumatic internal carotid artery thrombosis secondary to nonpenetrating injuries to the neck; a problem in the differential diagnosis of craniocerebral trauma. J. Neurosurg. 1952, 9, 495-507. [CrossRef] [PubMed]

16. Pitner, S.E. Carotid thrombosis due to intraoral trauma. An unusual complication of a common childhood accident. N. Engl. J. Med. 1966, 7, 764-767. [CrossRef] [PubMed]

17. Bor-Seng-Shu, E.; Hirsch, R.; Teixeira, M.J.; De Andrade, A.F.; Marino, R., Jr. Cerebral hemodynamic changes gauged by transcranial Doppler ultrasonography in patients with posttraumatic brain swelling treated by surgical decompression. J. Neurosurg. 2006, 104, 93-100. [CrossRef] [PubMed]

18. Tsunekawa, T.; Sawada, M.; Kato, T.; Motoji, Y.; Kinoshita, T.; Hirakawa, A.; Okawa, Y.; Tomita, S. The prevalence and distribution of occlusive lesions of the cerebral arteries in patients undergoing coronary artery bypass graft surgery. Semin. Thorac. Cardiovasc Surg. 2018, 30, 413-420. [CrossRef]

19. Pinho-Gomes, A.C.; Taggart, D.P. Coronary artery bypass grafting for left main disease and the risk of stroke: Incidence, aetiology and prevention. Surgeon 2017, 15, 155-160. [CrossRef]

20. Awad, E.; Asada, H.H. The Doppler Necklace: A wearable and noninvasive ultrasound sensor for continuous monitoring of blood flow in the common carotid artery. In Proceedings of the First Joint BMES/EMBS Conference. 1999 IEEE Engineering in Medicine and Biology 21st Annual Conference and the 1999 Annual Fall Meeting of the Biomedical Engineering Society (Cat. No.99CH37015), Atlanta, GA, USA, 13-16 October 1999; Volume 2, p. 795. [CrossRef]

21. Awad, E. Design of a Wearable Ultrasound Doppler Sensor to Monitor Blood Flow in the Common Carotid Artery. Master's Thesis, American University of Beirut, Lebanon, MA, USA, June 1999.

22. Pietrangelo, S.J. An Electronically Steered, Wearable Transcranial Doppler Ultrasound System; MIT: Lebanon, MA, USA, 2013.

23. Huang, C.C.; Lee, P.Y.; Chen, P.Y.; Liu, T.Y. Design and implementation of a smartphone-based portable ultrasound pulsed-wave Doppler device for blood flow measurement. IEEE Trans. Ultrason. Ferroelectr. Freq. Control. 2012, 59, 182-188. [CrossRef]

24. Shomaji, S.; Basak, A.; Mandai, S.; Karam, R.; Bhunia, S. A wearable carotid ultrasound assembly for early detection of cardiovascular diseases. In Proceedings of the 2016 IEEE Healthcare Innovation Point-Of-Care Technologies Conference (HI-POCT), Cancun, Mexico, 11-19 November 2016; pp. 17-20.

25. Taylor, K.J.; Holland, S. Doppler US. Part I. Basic principles, instrumentation, and pitfalls. Radiology 1990, 174, 297-307. [CrossRef] [PubMed] 
26. Quistgaard, J.U. Signal acquisition and processing in medical diagnostic ultrasound. IEEE Signal. Process. Mag. 1997, 14, 67-74. [CrossRef]

27. Vaitkus, P.J.; Cobbold, R.S. A comparative study and assessment of Doppler ultrasound spectral estimation techniques. Part I: Estimation methods. Ultrasound Med. Biol. 1988, 14, 661-672. [CrossRef]

28. Vaitkus, P.J.; Cobbold, R.S.; Johnston, K.W. A comparative study and assessment of Doppler ultrasound spectral estimation techniques. Part II: Methods and results. Ultrasound Med. Biol. 1988, 14, 673-688. [CrossRef]

29. Deng, G. A generalized unsharp masking algorithm. IEEE Trans. Image Process. 2011, 20, 1249-1261. [CrossRef] [PubMed]

(C) 2019 by the authors. Licensee MDPI, Basel, Switzerland. This article is an open access article distributed under the terms and conditions of the Creative Commons Attribution (CC BY) license (http://creativecommons.org/licenses/by/4.0/). 